\title{
Existence of Positive Solutions for a Third-Order Multi-Point Boundary Value Problem
}

\author{
A. Guezane-Lakoud, L. Zenkoufi \\ ${ }^{1}$ Laboratory of Advanced Materials, Faculty of Sciences, \\ University Badji Mokhtar-Annaba, Annaba, Algerie \\ ${ }^{2}$ Université 8 mai 1945 Guelma, Guelma, Algerie \\ Email: a_guezane@yahoo.fr, zenkoufi@yahoo.fr
}

Received June 25, 2012; revised July 25, 2012; accepted August 3, 2012

\begin{abstract}
By using Leray-Schauder nonlinear alternative, Banach contraction theorem and Guo-Krasnosel'skii theorem, we discuss the existence, uniqueness and positivity of solution to the third-order multi-point nonhomogeneous boundary value problem (BVP1):

$$
\left\{\begin{array}{l}
u^{\prime \prime \prime}+f\left(t, u(t), u^{\prime}(t)\right)=0, t \in(0,1) \\
u(0)=u^{\prime}(0)=0, u^{\prime}(1)=\sum_{i=1}^{n} \beta_{i} u^{\prime}\left(\eta_{i}\right)
\end{array}\right.
$$

where $f \in C\left([0,1] \times \mathrm{R}^{2}, \mathrm{R}\right), \quad \beta_{i}>0, \quad 0<\eta_{i}<1 ; \quad$ for $i=1, \cdots, n$. The interesting point lies in the fact that the nonlinear term is allowed to depend on the first order derivative $u^{\prime}$.

Keywords: Guo’s Fixed Point Theorem; Three Point Boundary Value Problem; Positive Solution; Leray Schauder Non-Linear Alternative; Contraction Principle
\end{abstract}

\section{Introduction}

It shows that problems related to nonlocal conditions have many applications in many problems such as in the theory of heat conduction, thermoelasticity, plasma physics, control theory, etc. The current analysis of these problems has a great interest and many methods are used to solve such problems. Recently certain three point boundary value problems for nonlinear ordinary differential equations have been studied by many authors [1-9]. The literature concerning these problems is extensive and application of theorems of functional analysis has attracted more interest. Recently, the study of existence of positive solution to third-order boundary value problems has gained much attention and is a rapidly growing field see [1,2,6,8-11]. However the approaches used in the literature are usually topological degree theory and fixed-point theorems in cone. We are interested in the existence, uniqueness and positivity of solution to the third-order multi-point nonhomogeneous boundary value problem (BVP1):

$$
\left\{\begin{array}{l}
u^{\prime \prime \prime}+f\left(t, u(t), u^{\prime}(t)\right)=0, t \in(0,1) \\
u(0)=u^{\prime}(0)=0, u^{\prime}(1)=\sum_{i=1}^{n} \beta_{i} u^{\prime}\left(\eta_{i}\right)
\end{array}\right.
$$

where $f \in C\left([0,1] \times \mathrm{R}^{2}, \mathrm{R}\right), \quad \beta_{i}>0, \quad 0<\eta_{i}<1 ; \quad$ for $i=1, \cdots, n$.

The organization of this paper is as follows. In Section 2, we present some preliminaries that will be used to prove our results. In Section 3, we discuss the existence and uniqueness of solution for the BVP1 by using Leray-Schauder nonlinear alternative and Banach contraction theorem. Finally, in Section 4 we study the positivity of solution by applying the Guo-Krasnosel'skii fixed point theorem.

\section{Preliminary Lemmas}

We first introduce some useful spaces. we will use the classical Banach spaces, $C[0,1], C^{1}[0,1], L^{1}[0,1]$. We also use the Banach space

$X=\left\{u \in C^{1}[0,1] / u \in C[0,1], u^{\prime} \in C[0,1]\right\}$, equipped with the norm $\|u\|_{X}=\max \left\{\|u\|_{\infty},\left\|u^{\prime}\right\|_{\infty}\right\}$, where

$\|u\|_{\infty}=\max _{t \in[0,1]}|u(t)|$.

Firstly we state some preliminary results.

Lemma 1 Let $\sum_{i=1}^{n} \beta_{i} \eta_{i} \neq 1$ and $y \in L^{1}[0,1]$, then the problem

$$
u^{\prime \prime \prime}+y(t)=0, \quad 0<t<1
$$




$$
u(0)=u^{\prime}(0)=0, u^{\prime}(1)=\sum_{i=1}^{n} \beta_{i} u^{\prime}\left(\eta_{i}\right),
$$

has a unique solution

$$
\begin{aligned}
u(t)= & \int_{0}^{1} G(t, s) y(s) \mathrm{d} s \\
& +\frac{t^{2}}{2\left(1-\sum_{i=1}^{n} \beta_{i} \eta_{i}\right)} \sum_{i=1}^{n} \beta_{i} \int_{0}^{1} G^{*}\left(\eta_{i}, s\right) y(s) \mathrm{d} s,
\end{aligned}
$$

where

$$
\begin{gathered}
G(t, s)=\frac{1}{2} \begin{cases}(1-s) t^{2}, & t \leq s \\
\left(-s+2 t-t^{2}\right) s, & s \leq t\end{cases} \\
G^{*}(t, s)=\frac{\partial G(t, s)}{\partial t}= \begin{cases}(1-s) t, & t \leq s \\
(1-t) s, & s \leq t .\end{cases}
\end{gathered}
$$

Proof Integrating the Equation (2.1), it yields

$$
u(t)=-\frac{1}{2} \int_{0}^{t}(t-s)^{2} y(s) \mathrm{d} s+\frac{1}{2} C_{1} t^{2}+C_{2} t+C_{3} .
$$

From the boundary condition $u(0)=u^{\prime}(0)=0$, we deduce that $C_{3}=0$, and $C_{2}=0$.

And the boundary condition $u^{\prime}(1)=\sum_{i=1}^{n} \beta_{i} u^{\prime}\left(\eta_{i}\right)$, implies

$$
\begin{aligned}
C_{1}= & \frac{1}{1-\sum_{i=1}^{n} \beta_{i} \eta_{i}} \int_{0}^{1}(1-s) y(s) \mathrm{d} s \\
& -\frac{\sum_{i=1}^{n} \beta_{i}}{1-\sum_{i=1}^{n} \beta_{i} \eta_{i}} \int_{0}^{\eta_{i}}\left(\eta_{i}-s\right) y(s) \mathrm{d} s .
\end{aligned}
$$

Therefore we have

$$
\begin{aligned}
u(t)= & -\frac{1}{2} \int_{0}^{t}(t-s)^{2} y(s) d s+\frac{t^{2}}{2} \int_{0}^{1}(1-s) y(s) \mathrm{d} s \\
& +\frac{\sum_{i=1}^{n} \beta_{i} \eta_{i} t^{2}}{2\left(1-\sum_{i=1}^{n} \beta_{i} \eta_{i}\right)} \int_{0}^{1}(1-s) y(s) \mathrm{d} s \\
& -\frac{\sum_{i=1}^{n} \beta_{i} t^{2}}{2\left(1-\sum_{i=1}^{n} \beta_{i} \eta_{i}\right)} \int_{0}^{\eta_{i}}\left(\eta_{i}-s\right) y(s) \mathrm{d} s .
\end{aligned}
$$

Now it is easy to have

$$
\begin{aligned}
u(t)= & \int_{0}^{1} G(t, s) y(s) \mathrm{d} s \\
& +\frac{t^{2}}{2\left(1-\sum_{i=1}^{n} \beta_{i} \eta_{i}\right)} \sum_{i=1}^{n} \beta_{i} \int_{0}^{1} G^{*}\left(\eta_{i}, s\right) y(s) \mathrm{d} s .
\end{aligned}
$$

which achieves the proof of Lemma 1.

We need some properties of functions $G(t, s)$.

Lemma 2 For all $t, s$, such that $0 \leq s, t \leq 1$, we have

$$
0 \leq G^{*}(t, s) \leq 2 G(1, s)
$$

Proof It is easy to see that, if $t \leq s$,
$G^{*}(t, s)=(1-s) t \leq(1-s) s$. If $s \leq t$ then $G^{*}(t, s)=(1-t) s \leq(1-s) s=2 G(1, s)$.

Lemma 3 For all $t, s$ such that $0 \leq s \leq 1,0<\tau \leq t \leq 1$, we have

$$
\tau^{2} G(1, s) \leq G(t, s) \leq G(1, s)=\frac{1}{2}(1-s) s,
$$

Proof For all $t, s \in[0,1]$, if $s \leq t$, it follows from (2.4) that

$$
\begin{aligned}
G(t, s) & =\frac{1}{2}\left(2 t-t^{2}-s\right) s=\frac{1}{2}\left[(1-s)-(1-t)^{2}\right] s \\
& \leq \frac{1}{2}(1-s) s=G(1, s),
\end{aligned}
$$

and

$$
\begin{aligned}
G(t, s) & =\frac{1}{2}\left(2 t-t^{2}-s\right) s \\
& =\frac{1}{2} s t^{2}(1-s)+\frac{1}{2}(1-t)[(t-s)+(1-s) t] s \\
& \geq t^{2} G(1, s) .
\end{aligned}
$$

If $t \leq s$, it follows from (2.4) that

$$
\frac{1}{2} t^{2}(1-s) s \leq G(t, s)=\frac{1}{2} t^{2}(1-s) \leq G(1, s) .
$$

Therefore

$$
\tau^{2} G(1, s) \leq G(t, s) \leq G(1, s), \forall(t, s) \in[\tau, 1] \times[0,1]
$$

Lemma 4 (See [6]) We define an operator $T: X \rightarrow X$ by

$$
\begin{aligned}
T u(t) & =\int_{0}^{1} G(t, s) f\left(s, u(s), u^{\prime}(s)\right) \mathrm{d} s \\
& +\frac{t^{2} \sum_{i=1}^{n} \beta_{i}}{2\left(1-\sum_{i=1}^{n} \beta_{i} \eta_{i}\right)} \int_{0}^{1} G^{*}\left(\eta_{i}, s\right) f\left(s, u(s), u^{\prime}(s)\right) \mathrm{d} s .
\end{aligned}
$$

Lemma 5 (See [5]) The function $u \in E$ is a solution of the (BVP1) if and only if $T$ has a fixed point in $X$, i.e. $T u(t)=u(t)$.

\section{Existence Results}

Now, we give some existence results for the BVP1

Theorem 6 Assume that $\sum_{i=1}^{n} \beta_{i} \eta_{i} \neq 1$ and there exist nonnegative functions $k, h \in L^{1}\left([0,1], R_{+}\right)$, such that $\forall x, y, u, v \in R, \quad t \in[0,1]$ we have

$$
|f(t, x, y)-f(t, u, v)| \leq k(t)|x-u|+h(t)|y-v|,
$$

and

$$
\int_{0}^{1} G(1, s)(k(s)+h(s)) \mathrm{d} s<\frac{1-\sum_{i=1}^{n} \beta_{i} \eta_{i}}{2\left(1+\sum_{i=1}^{n} \beta_{i}\left(1-\eta_{i}\right)\right)}
$$

then, the (BVP1) has a unique solution in $X$.

Proof We shall prove that $T$ is a contraction. Let $u, v \in X$, then 


$$
\begin{aligned}
|T u(t)-T v(t)| \leq & \int_{0}^{1} G(1, s)\left|f\left(s, u(s), u^{\prime}(s)\right)-f\left(s, v(s), v^{\prime}(s)\right)\right| \mathrm{d} s \\
& +\frac{\sum_{i=1}^{n} \beta_{i}}{1-\sum_{i=1}^{n} \beta_{i} \eta_{i}} \int_{0}^{1} G(1, s)\left|f\left(s, u(s), u^{\prime}(s)\right)-f\left(s, v(s), v^{\prime}(s)\right)\right| \mathrm{d} s,
\end{aligned}
$$

So we can obtain

$$
\begin{aligned}
|T u(t)-T v(t)| & \leq\left(1+\frac{\sum_{i=1}^{n} \beta_{i}}{1-\sum_{i=1}^{n} \beta_{i} \eta_{i}}\right) \max _{0 \leq t \leq 1}\left\{\|u-v\|_{\infty},\left\|u^{\prime}-v^{\prime}\right\|_{\infty}\right\} \times \int_{0}^{1} G(1, s)(k(s)+h(s)) \mathrm{d} s, \\
& \leq\|u-v\|_{X} .
\end{aligned}
$$

Similarly, we have

$$
\begin{aligned}
\left|T^{\prime} u(t)-T^{\prime} v(t)\right| & \leq 2\left(1+\frac{\sum_{i=1}^{n} \beta_{i}}{1-\sum_{i=1}^{n} \beta_{i} \eta_{i}}\right) \max _{0 \leq t \leq 1}\left\{\|u-v\|_{\infty},\left\|u^{\prime}-v^{\prime}\right\|_{\infty}\right\} \times \int_{0}^{1} G(1, s)(k(s)+h(s)) \mathrm{d} s, \\
& \leq\|u-v\|_{X}
\end{aligned}
$$

From this we deduce

$$
\|T u-T v\|_{X} \leq\|u-v\|_{X} .
$$

Then $T$ is a contraction. From Banach contraction principe we deduce that $T$ has a unique fixed point which is the unique solution of (BVP1).

We will employ the following Leray-Schauder nonlinear alternative [12].

Lemma 7 Let Fbe Banach space and $\Omega$ be a bounded open subset of $F, 0 \in \Omega . T: \bar{\Omega} \rightarrow F$ be a completely continuous operator. Then, either there exists $x \in \partial \Omega$, $\lambda>1$ such that $T(x)=\lambda x$, or there exists a fixed point $x^{*} \in \bar{\Omega}$.

Theorem 8 We assume that $f(t, 0,0) \neq 0$, $\sum_{i=1}^{n} \beta_{i} \eta_{i} \neq 1$ and there exist nonnegative functions $k, l, h \in L^{1}[0,1]$ such that

$$
\begin{gathered}
|f(t, u, v)| \leq k(t)|u|+l(t)|v|+h(t), \quad \forall u, v \in R, t \in[0,1], \\
2\left(1+\frac{\sum_{i=1}^{n} \beta_{i}}{1-\sum_{i=1}^{n} \beta_{i} \eta_{i}}\right) \int_{0}^{1} G(1, s)(k(s)+l(s)) \mathrm{d} s<1
\end{gathered}
$$

Then the (BVP1) has at least one nontrivial solution $u^{*} \in X$.

Proof Setting

$$
\begin{aligned}
& F=2\left(1+\frac{\sum_{i=1}^{n} \beta_{i}}{1-\sum_{i=1}^{n} \beta_{i} \eta_{i}}\right) \int_{0}^{1} G(1, s)(k(s)+l(s)) \mathrm{d} s, \\
& G=2\left(1+\frac{\sum_{i=1}^{n} \beta_{i}}{1-\sum_{i=1}^{n} \beta_{i} \eta_{i}}\right) \int_{0}^{1} G(1, s) h(s) \mathrm{d} s .
\end{aligned}
$$

Remarking that $F<1 . \quad f(t, 0,0) \neq 0$ and $G>0$, then there exists an interval $[\sigma, r] \subset[0,1]$ such that $\min _{\sigma \leq t \leq r}|f(t, 0,0)|>0$ and $h(t) \geq|f(t, 0,0)|$, a.e. $t \in[0,1]$.
Le $m=G(1-F)^{-1}, \quad \Omega=\{u \in X:\|u\|<m\}$. With the help of Ascoli-Arzela Theorem we show that $T: \bar{\Omega} \rightarrow X$ is a completely continuous mapping. We assume that $u \in \partial \Omega, \quad \lambda>1$ such that $T u=\lambda u$, then $\lambda m=\lambda\|u\|=\|T u\|_{X}=\max _{0 \leq t \leq 1}\left\{\|T u\|_{\infty},\left\|T^{\prime} u\right\|_{\infty}\right\}$, we have

$$
\begin{aligned}
|T u(t)| \leq & \|u\|_{X}\left(1+\frac{\sum_{i=1}^{n} \beta_{i}}{1-\sum_{i=1}^{n} \beta_{i} \eta_{i}}\right) \int_{0}^{1} G(1, s)(k(s)+l(s)) \mathrm{d} s \\
& +\left(1+\frac{\sum_{i=1}^{n} \beta_{i}}{1-\sum_{i=1}^{n} \beta_{i} \eta_{i}}\right) \int_{0}^{1} G(1, s) h(s) \mathrm{d} s
\end{aligned}
$$

and

$$
\begin{aligned}
& \left|T^{\prime} u(t)\right| \\
& \leq 2\|u\|_{X}\left(1+\frac{\sum_{i=1}^{n} \beta_{i}}{1-\sum_{i=1}^{n} \beta_{i} \eta_{i}}\right) \int_{0}^{1} G(1, s)(k(s)+l(s)) \mathrm{d} s \\
& +2\left(1+\frac{\sum_{i=1}^{n} \beta_{i}}{1-\sum_{i=1}^{n} \beta_{i} \eta_{i}}\right) \int_{0}^{1} G(1, s) h(s) \mathrm{d} s .
\end{aligned}
$$

This shows that $\lambda m=\|T u\|_{X} \leq F\|u\|_{X}+G=F m+G$. From this we get

$$
\lambda \leq F+\frac{G}{m}=F+\frac{G}{G(1-F)^{-1}}=F+(1-F)=1,
$$

this contradicts $\lambda>1$. By applying Lemma $7, T$ has a fixed point $u^{*} \in \bar{\Omega}$ and then the BVP1 has a nontrivial solution $u^{*} \in X$.

\section{Positive Results}

In this section, we discuss the existence of positive solutions for (BVP1). We make the following additional as- 
sumptions.

(Q1) $f(t, u, v)=a(t) f_{1}(u, v)$ where $a \in C\left([0,1], \mathrm{R}_{+}\right)$ and $f_{1} \in C\left(\mathrm{R}_{+} \times \mathrm{R}, \mathrm{R}_{+}\right)$.

(Q2) $\int_{0}^{1} G(1, s) a(s) \mathrm{d} s>0$.

We need some properties of functions $G(t, s)$.

Lemma 9 For all $0<\tau_{1} \leq t \leq \tau_{2}<1, s \in[0,1]$, we have

$$
\tau_{1}^{2} G(1, s) \leq G(t, s),
$$

$2 \gamma G(1, s) \leq G^{*}(t, s)$ where $\gamma=\min \left\{\tau_{1}^{2},\left(1-\tau_{2}\right)\right\}$.

Proof It is easy to see that.

If $t \leq s$,

$$
\begin{aligned}
G^{*}(t, s) & =(1-s) t=(1-s) s \frac{t}{s} \\
& \geq(1-s) s \tau_{1} \geq 2 \tau_{1}^{2} G(1, s) .
\end{aligned}
$$

If $s \leq t \Rightarrow-t \leq-s$,

$$
\begin{aligned}
G^{*}(t, s) & =(1-t) s=\frac{1-t}{1-s}(1-s) s \\
& \geq\left(1-\tau_{2}\right)(1-s) s=2\left(1-\tau_{2}\right) G(1, s) .
\end{aligned}
$$

Lemma 10 Let $u \in X$ and assume that $0<\sum_{i=1}^{n} \beta_{i} \eta_{i}<1$, then the unique solution $u$ of the (BVP1) is nonnegative and satisfies

$$
\min _{t \in\left[\tau_{1}, \tau_{2}\right]}\left(u(t)+u^{\prime}(t)\right) \geq \gamma\left(1+\frac{\sum_{i=1}^{n} \beta_{i}}{\sum_{i=1}^{n} \beta_{i} \eta_{i}}\right)^{-1}\|u\|_{X},
$$

Proof Let $u \in X$, it is obvious that $u(t)$ is nonnegative. For any $t \in[0,1]$, by (2.3) and Lemmas 2 and 3 , it follows that

$$
\begin{aligned}
& \|u\|_{\infty} \\
& \leq\left(1+\frac{\sum_{i=1}^{n} \beta_{i}}{1-\sum_{i=1}^{n} \beta_{i} \eta_{i}}\right) \int_{0}^{1} G(1, s) a(s) f_{1}\left(u(s), u^{\prime}(s)\right) \mathrm{d} s .
\end{aligned}
$$

On the other hand, (2.4) and Lemma 11 imply that, for any $t \in\left[\tau_{1}, \tau_{2}\right]$, we have

$$
\begin{aligned}
u(t) & \geq \int_{0}^{1} G(t, s) a(s) f_{1}\left(u(s), u^{\prime}(s)\right) \mathrm{d} s \\
& \geq \tau_{1}^{2} \int_{0}^{1} G(1, s) a(s) f_{1}\left(u(s), u^{\prime}(s)\right) \mathrm{d} s
\end{aligned}
$$

From (4.1) it yields

$$
u(t) \geq \tau_{1}^{2}\left(1+\frac{\sum_{i=1}^{n} \beta_{i}}{1-\sum_{i=1}^{n} \beta_{i} \eta_{i}}\right)^{-1}\|u\|_{\infty}
$$

Therefore, we have

$$
\min _{t \in\left[\tau_{1}, \tau_{2}\right]} u(t) \geq \tau_{1}^{2}\left(1+\frac{\sum_{i=1}^{n} \beta_{i}}{1-\sum_{i=1}^{n} \beta_{i} \eta_{i}}\right)^{-1}\|u\|_{\infty}
$$

Similarly, we get

$\left\|u^{\prime}\right\|_{\infty}$

$$
\leq 2\left(1+\frac{\sum_{i=1}^{n} \beta_{i}}{1-\sum_{i=1}^{n} \beta_{i} \eta_{i}}\right) \int_{0}^{1} G(1, s) a(s) f_{1}\left(u(s), u^{\prime}(s)\right) \mathrm{d} s
$$

On the other hand, for $0<\tau_{1} \leq t \leq \tau_{2}<1$ and using Lemma 10 and (4.1) we obtain

$$
\begin{aligned}
& u^{\prime}(t) \geq \int_{0}^{1} 2 \gamma G(1, s) a(s) f_{1}\left(u(s), u^{\prime}(s)\right) \mathrm{d} s \\
& \geq \gamma\left(1+\frac{\sum_{i=1}^{n} \beta_{i}}{1-\sum_{i=1}^{n} \beta_{i} \eta_{i}}\right)^{-1}\left\|u^{\prime}\right\|_{\infty}
\end{aligned}
$$

Therefore,

$$
\min _{t \in\left[\tau_{1}, \tau_{2}\right]} u^{\prime}(t) \geq \gamma\left(1+\frac{\sum_{i=1}^{n} \beta_{i}}{1-\sum_{i=1}^{n} \beta_{i} \eta_{i}}\right)^{-1}\left\|u^{\prime}\right\|_{\infty}
$$

Finally, regrouping (4.2) and (4.3) we have

$$
\min _{t \in\left[\tau_{1}, \tau_{2}\right]}\left(u(t)+u^{\prime}(t)\right) \geq \gamma\left(1+\frac{\sum_{i=1}^{n} \beta_{i}}{1-\sum_{i=1}^{n} \beta_{i} \eta_{i}}\right)^{-1}\|u\|_{X}
$$

Definition 11 Let use introduce the following sets

$$
\begin{aligned}
& K=\{u \in X, u(t) \geq 0, \\
& \left.\min _{t \in\left[\tau_{1}, \tau_{2}\right]}\left(u(t)+u^{\prime}(t)\right) \geq \gamma\left(1+\frac{\sum_{i=1}^{n} \beta_{i}}{1-\sum_{i=1}^{n} \beta_{i} \eta_{i}}\right)^{-1}\|u\|_{X}\right\}
\end{aligned}
$$

$K$ is a non-empty closed and convex subset of $X$.

Lemma 12 (See [5]) The operator $T$ is completely continuous and satisfies $T(K) \subseteq K$.

To establish the existence of positive solutions of (BVP1), we will use the following Guo-Krasnosel'skii fixed point theorem [13].

Theorem 13 Let $E$ be a Banach space and let $K \subset E$, 
be a cone. Assume that $\Omega_{1}, \Omega_{2}$ are open subsets of $E$ with $0 \in \Omega_{1}, \quad \bar{\Omega}_{1} \subset \Omega_{2}$, and let

$$
\mathrm{A}: K \cap\left(\bar{\Omega}_{2} \backslash \Omega_{1}\right) \rightarrow K
$$

be a completely continuous operator. In addition suppose either

1) $\|A u\| \leq\|u\|, \quad u \in K \cap \partial \Omega_{1}$, and $\|A u\| \geq\|u\|$, $u \in K \cap \partial \Omega_{2}$; or

2) $\|\mathrm{A} u\| \geq\|u\|, \quad u \in K \cap \partial \Omega_{1}$, and $\|\mathrm{A} u\| \leq\|u\|$, $u \in K \cap \partial \Omega_{2}$.

holds. Then A has a fixed point in $K \cap\left(\bar{\Omega}_{2} \backslash \Omega_{1}\right)$.

The main result of this section is the following

Theorem 14 Let $\left(Q_{1}\right)$ and $\left(Q_{2}\right)$ hold, $0 \leq \sum_{i=1}^{n} \beta_{i} \eta_{i}<1$ and assume that

$$
f_{0}=\lim _{(|u|+|v|) \rightarrow 0} \frac{f_{1}(u, v)}{|u|+|v|}, f_{\infty}=\lim _{(|u|+v \mid) \rightarrow \infty} \frac{f_{1}(u, v)}{|u|+|v|} .
$$

Then the problem (BVP1) has at least one positive solution in the case

1) $f_{0}=0$ and $f_{\infty}=\infty$ (superlinear) or

2) $f_{0}=\infty$ and $f_{\infty}=0$ (sublinear).

Proof We prove the superlinear case. Since $f_{0}=0$, then for any $\varepsilon>0, \exists \delta_{1}>0$, such that

$f_{1}(u, v) \leq \varepsilon(|u|+|v|)$, for $|u|+|v|<\delta_{1}$. Let $\Omega_{1}$ be an open set in $X$ defined by

$$
\Omega_{1}=\left\{y \in X /\|y\|<\delta_{1}\right\}
$$

then, for any $u \in K \cap \partial \Omega_{1}$, it yields

$$
T u(t) \leq \varepsilon\|u\|_{X}\left(1+\frac{\sum_{i=1}^{n} \beta_{i}}{1-\sum_{i=1}^{n} \beta_{i} \eta_{i}}\right) \int_{0}^{1} G(1, s) a(s) \mathrm{d} s
$$

Therefore

$$
\|T u(t)\|_{\infty} \leq \varepsilon\|u\|_{X}\left(1+\frac{\sum_{i=1}^{n} \beta_{i}}{1-\sum_{i=1}^{n} \beta_{i} \eta_{i}}\right) \int_{0}^{1} G(1, s) a(s) \mathrm{d} s
$$

So

$$
\left\|T^{\prime} u(t)\right\|_{\infty} \leq 2 \varepsilon\|u\|_{X}\left(1+\frac{\sum_{i=1}^{n} \beta_{i}}{1-\sum_{i=1}^{n} \beta_{i} \eta_{i}}\right) \int_{0}^{1} G(1, s) a(s) \mathrm{d} s
$$

If we choose

$$
\varepsilon=\left[3\left(1+\frac{\sum_{i=1}^{n} \beta_{i}}{1-\sum_{i=1}^{n} \beta_{i} \eta_{i}}\right) \int_{0}^{1} G(1, s) a(s) \mathrm{d} s\right]^{-1},
$$

then it yields

$$
\|T u\|_{X} \leq\|u\|_{X}, \forall u \in K \cap \partial \Omega_{1} .
$$

Now from $f_{\infty}=\infty$, we have $\forall M>0, \quad \exists H>0$, such that $f_{1}(u, v) \geq M(|u|+|v|)$ for $|u|+|v| \geq H$. Let $H_{1}=\max \left\{2 \delta_{1}, \frac{H}{\gamma}\right\}$. Denote by $\Omega_{2}$ the open set
$\Omega_{2}=\left\{y \in X /\|y\|<H_{1}\right\}$.

If $u \in K \cap \partial \Omega_{2}$ then

$$
\min _{t \in\left[\tau_{1}, \tau_{2}\right]}\left\{u(t), u^{\prime}(t)\right\} \geq \gamma\|u\|_{X}=\gamma H_{1} \geq H .
$$

then $\bar{\Omega}_{1} \subset \Omega_{2}$. Let $u \in K \cap \partial \Omega_{2}$ then

$$
T u(t) \geq\|u\|_{X} M \gamma\left(1+\frac{\sum_{i=1}^{n} \beta_{i}}{1-\sum_{i=1}^{n} \beta_{i} \eta_{i}}\right) \int_{0}^{1} G(1, s) a(s) \mathrm{d} s .
$$

And

$$
T^{\prime} u(t) \geq\|u\|_{X} M \gamma\left(1+\frac{\sum_{i=1}^{n} \beta_{i}}{1-\sum_{i=1}^{n} \beta_{i} \eta_{i}}\right) \int_{0}^{1} G(1, s) a(s) \mathrm{d} s
$$

Choosing

$$
M=\left[\gamma\left(1+\frac{\sum_{i=1}^{n} \beta_{i}}{1-\sum_{i=1}^{n} \beta_{i} \eta_{i}}\right) \int_{0}^{1} G(1, s) a(s) \mathrm{d} s\right]^{-1},
$$

we get $\|T u\|_{X} \geq\|u\|_{X}, \quad \forall u \in K \cap \partial \Omega_{2}$. By the first part of Theorem13, $T$ has at least one fixed point in

$K \cap\left(\bar{\Omega}_{2} \backslash \Omega_{1}\right)$ such that $H \leq\|y\| \leq H_{1}$. This completes the superlinear case of the theorem 14. Proceeding as above we proof the sublinear case. This achieves the proof of Theorem 14.

Example 15 Consider the following boundary value problem

$$
\left\{\begin{array}{l}
u^{\prime \prime \prime}+t^{2}+4+\frac{2 t}{3} u \sin u+\frac{1}{5}(t+1) u^{\prime}=0,0<t<1 \\
u(0)=u^{\prime}(0)=0, \quad u^{\prime}(1)=\sum_{i=1}^{3} \beta_{i} u^{\prime}\left(\eta_{i}\right) .
\end{array}\right.
$$

Set $\quad \beta_{1}=\frac{1}{3}, \quad \beta_{2}=\frac{1}{4}, \quad \beta_{3}=\frac{1}{5} \quad$ and $\quad \eta_{1}=\frac{1}{4}, \quad \eta_{2}=\frac{1}{3}$, $\eta_{3}=\frac{1}{2}$ where $\sum_{i=1}^{3} \beta_{i} u^{\prime}\left(\eta_{i}\right)=\frac{16}{60}<1$ and and $f(t, u, v)=t^{2}+4+\frac{2 t}{3} u \sin u+\frac{1}{5}(t+1) v$. One can choose

$$
\left\{\begin{array}{l}
k(t)=\frac{2}{3} t \\
h(t)=\frac{(t+1)}{5}
\end{array}, t \in[0,1]\right.
$$

It is easy to prove that $k, \quad l \in L^{1}[0,1]$ are nonnegative functions, and

$$
\begin{aligned}
|f(t, x, y)-f(t, u, v)| & \leq \frac{2 t}{3}|x-u|+\frac{(t+1)}{5}|y-v|, \\
& \leq k(t)|x-u|+h(t)|y-v| .
\end{aligned}
$$

Hence, by Theorem 6, the boundary value problem (E1) has a unique solution in $X$.

2) Now if we estimate $f$ as 


$$
\begin{aligned}
|f(t, u, v)| & \leq \frac{2 t}{3}|u|+\frac{(t+1)}{5}|v|+t^{2}+4, \\
& \leq k(t)|u|+l(t)|v|+h(t) .
\end{aligned}
$$

then one can choose $h(t)=t^{2}+4, t \in[0,1]$. So $k, l$, $h \in L^{1}[0,1]$ are nonnegative functions. Hence, by Theorem 8 , the boundary value problem (E1) has at least one nontrivial solution, $u^{*} \in X$.

Example 16 Consider the following boundary value problem

$$
\left\{\begin{array}{l}
u^{\prime \prime \prime}+t^{2} u^{2}+\frac{t^{2}}{5}\left(u^{\prime}\right)^{2}=0, \quad 0<t<1 \\
u(0)=u^{\prime}(0)=0, \quad u^{\prime}(1)=\sum_{i=1}^{3} \beta_{i} u^{\prime}\left(\eta_{i}\right) .
\end{array}\right.
$$

where, $0<\eta_{i}<1, \quad 0<\sum_{i=1}^{3} \beta_{i}<1$ and

$$
f(t, u, v)=t^{2}\left(u^{2}+\frac{1}{7} v^{2}\right)=a(t) f_{1}(u, v)
$$

Then $a(t)=t^{2} \in C\left((0,1), R_{+}\right)$,

$f_{1}(u, v) \in C\left(R_{+} \times R, R_{+}\right)$. We put, $u=r \cos \varphi$ and $v=r \sin \varphi$, when $(|u|+|v|) \rightarrow 0 \Rightarrow r \rightarrow 0$ and when $(|u|+|v|) \rightarrow \infty \Rightarrow r \rightarrow \infty$

Then

$$
\begin{aligned}
& f_{0}=\lim _{(|u+| v \mid) \rightarrow 0} \frac{f_{1}(u, v)}{|u|+|v|}=0 \\
& f_{\infty}=\lim _{(|u|+|v|) \rightarrow \infty} \frac{f_{1}(u, v)}{|u|+|v|}=\infty
\end{aligned}
$$

By theorem 13 1) the BVP (E2) has at least one positive solution.

\section{REFERENCES}

[1] D. Anderson and R. Avery, "Multiple Positive Solutions to Third-Order Discrete Focal Boundary Value Problem," Acta Mathematicae Applicatae Sinica, Vol. 19, No. 1, 2003, pp. 117-122. doi:10.1007/s10255-003-0087-1

[2] D. R. Anderson, “Green's Function for a Third-Order
Generalized Right Focal Problem,” Journal of Mathematical Analysis and Applications, Vol. 288, No. 1, 2003, pp. 1-14. doi:10.1016/S0022-247X(03)00132-X

[3] A. Guezane-Lakoud and L. Zenkoufi, "Positive Solution of a Three-Point Nonlinear Boundary Value Problem for Second Order Differential Equations,” International Journal of Applied Mathematics and Statistics, Vol. 20, 2011, pp. 38-46.

[4] A. Guezane-Lakoud and S. Kelaiaia, "Solvability of a Three-Point Nonlinear Noundary-Value Problem," Electronic Journal of Differential Equations, Vol. 2010, No. 139, 2010, pp. 1-9.

[5] A. Guezane-Lakoud, S. Kelaiaia and A. M. Eid, "A Positive Solution for a Non-Local Boundary Value Problem," International Journal of Open Problems in Computer Science and Mathematics, Vol. 4, No. 1, 2011, pp. 36-43.

[6] J. R. Graef and B. Yang, "Existence and Nonexistence of Positive Solutions of a Nonlinear Third Order Boundary Value Problem," Electronic Journal of Qualitative Theory of Differential Equations, No. 9, 2008, pp. 1-13.

[7] J. R. Graef and B. Yang, "Positive Solutions of a Nonlinear Third Order Eigenvalue Problem,” Dynamic Systems \& Applications, Vol. 15, 2006, pp. 97-110.

[8] S. Li, "Positive Solutions of Nonlinear Singular ThirdOrder Two-Point Boundary Value Problem,” Journal of Mathematical Analysis and Applications, Vol. 323, No. 1, 2006, pp. 413-425. doi:10.1016/j.jmaa.2005.10.037

[9] B. Hopkins and N. Kosmatov, "Third-Order Boundary Value Problems with Sign-Changing Solutions,” Nonlinear Analysis, Vol. 67, No. 1, 2007, pp. 126-137. doi:10.1016/j.na.2006.05.003

[10] L. J. Guo, J. P. Sun and Y. H. Zhao, "Existence of Positive Solutions for Nonlinear Third-Order Three-Point Boundary Value Problem,” Nonlinear Analysis, Vol. 68, No. 10, 2008, pp. 3151-3158. doi:10.1016/j.na.2007.03.008

[11] Y. Sun, "Positive Solutions of Singular Third-Order ThreePoint Boundary Value Problem,” Journal of Mathematical Analysis and Applications, Vol. 306, No. 2, 2005, pp. 589-603. doi:10.1016/j.jmaa.2004.10.029

[12] K. Deimling, "Nonlinear Functional Analysis,” Springer, Berlin, 1985. doi:10.1007/978-3-662-00547-7

[13] D. Guo and V. Lakshmikantham, "Nonlinear Problems in Abstract Cones,” Academic Press, San Diego, 1988. 